\title{
A Case of Popliteal Artery Aneurysm Associated with Popliteal Artery Entrapment Syndrome
}

\author{
Hiroichiro Yamaguchi, MD, ${ }^{1}$ Takashi Miura, MD, ${ }^{2}$ Kiyoyuki Eishi, MD, ${ }^{2}$ and Nobuo Tsuda, $\mathrm{MD}^{3}$
}

Popliteal artery entrapment (PAE) is an uncommon syndrome. Although this phenomenon can cause claudication in younger populations, other clinical features may vary. The present report describes a case of a 48-year-old woman who was referred for treatment of a pulsatile mass in the right popliteal fossa. Computed tomography scan and magnetic resonance imaging demonstrated popliteal aneurysm associated with PAE. Resection of abnormal musculotendinous structures, aneurysmectomy and subsequent revascularization were successfully performed. Although PAE may lead to poststenotic dilatation and aneurysm formation, huge aneurysm without clinical signs of ischemia in a patient with PAE is extremely rare.

Key words: popliteal artery entrapment syndrome, popliteal artery aneurysm

\section{INTRODUCTION}

$\mathrm{P}$ opliteal artery entrapment (PAE) syndrome is caused by a congenitally anomalous relationship between the popliteal artery and the musculotendinous structures of the popliteal space. Abnormal lateral attachment of the medial head of the gastrocnemius muscle with associated accessory muscle slips results in external popliteal artery compression. Claudication with strenuous exercise, particularly in younger patients, should prompt an evaluation for potential PAE. Although patients with PAE typically present with limb ischemia due to stenotic lesions of the popliteal artery, repetitive mechanical trauma to the artery can also lead to progressive fibrosis of the entrapped vessel wall and subsequent the poststenotic dilatation and the aneurysm formation. We report a case of popliteal

\footnotetext{
${ }^{1}$ Department of Cardiovascular Surgery, Nagasaki Municipal Medical Center, Nagasaki, Japan

${ }^{2}$ Division of Cardiovascular Surgery, Nagasaki University Graduate School of Biomedical Sciences, Nagasaki, Japan

${ }^{3}$ Department of Pathology, Nagasaki Prefecture Medical Health Operating Group, Nagasaki, Japan
}

Received: October 8, 2008 Accepted: January 27, 2010 Address for reprint requests to Hiroichiro Yamaguchi, MD: Department of Cardiovascular Surgery, Nagasaki Municipal Center, 20-5 Fuchi-machi, Nagasaki 852-8012, Japan

Tel: +81-095-861-1111, Fax: +81-095-861-8295

E-mail: spf85aq9@kind.ocn.ne.jp artery aneurysm associated with PAE.

\section{Case}

A 48-year-old female was referred with a 3-month progressive history of a pulsatile mass in the right popliteal fossa. Past medical history was unremarkable, and the patient denied any traumatic events. Physical examination revealed no particular abnormality other than the popliteal mass. Lower extremity pulses were normal at rest but weakened with passive dorsiflexion of the right foot. Ankle brachial pressure index (ABI) on the right lower extremity was 0.93 in the normal position and 0.65 with passive dorsiflexion.

Computed tomography (CT) scan showed a saccular popliteal aneurysm of $50 \mathrm{~mm}$ in diameter with mural thrombus in the right popliteal fossa (Fig. 1). A muscular structure was situated between the popliteal artery and the vein originating at the posterior surface of the medial epicondyle, and the proximal site of the popliteal artery deviated medially as a result of compression. PAE syndrome was suspected and confirmed by magnetic resonance imaging (MRI). The abnormal muscular structure on CT scan was identified as an accessory slip of the medial head of the gastrocnemius muscle, which deviated in the superiolateral direction, and a part of medial head of gastrocnemius muscle, which originated at a normal position 

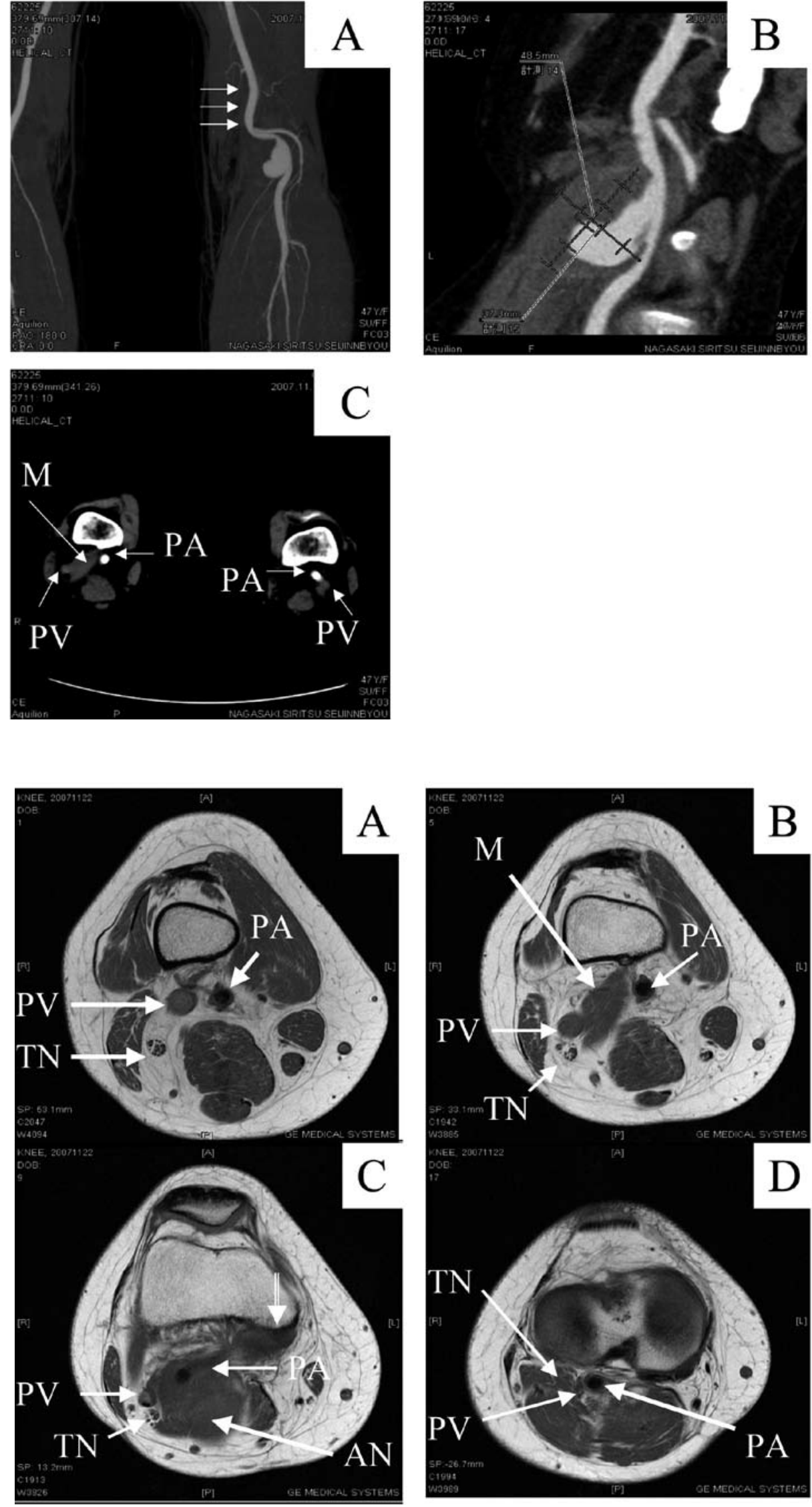

Fig. 1 Preoperative CT scan findings.

A: Entraped proximal site of the popliteal artery.

B: Saccular popliteal aneurysm of $50 \mathrm{~mm}$ in diameter with mural thrombus in the right popliteal fossa.

C: M: Accessory slip of the medial head of the gastrocnemius muscle, PA: popliteal artery, PV:popliteal vein.

Fig. 2 Preoperative MRI findings.

A: Popliteal artery in parallel with popliteal vein behind distal femur.

B: Accessory slip of the medial head of the gastrocnemius muscle between popliteal artery and vein.

C: Saccular popliteal aneurysm associated with entraped popliteal artery.

A part of medial head of gastrocnemius muscle, which originated at a normal position from the posterior surface of the medial epicondyle.

D: Popliteal artery in parallel with popliteal vein behind proximal tibia.

M: Accessory slip of the medial head of the gastrocnemius muscle, PA: popliteal artery, PV: popliteal vein, $\mathrm{TN}$ : tibial nerve, AN: aneurysm 

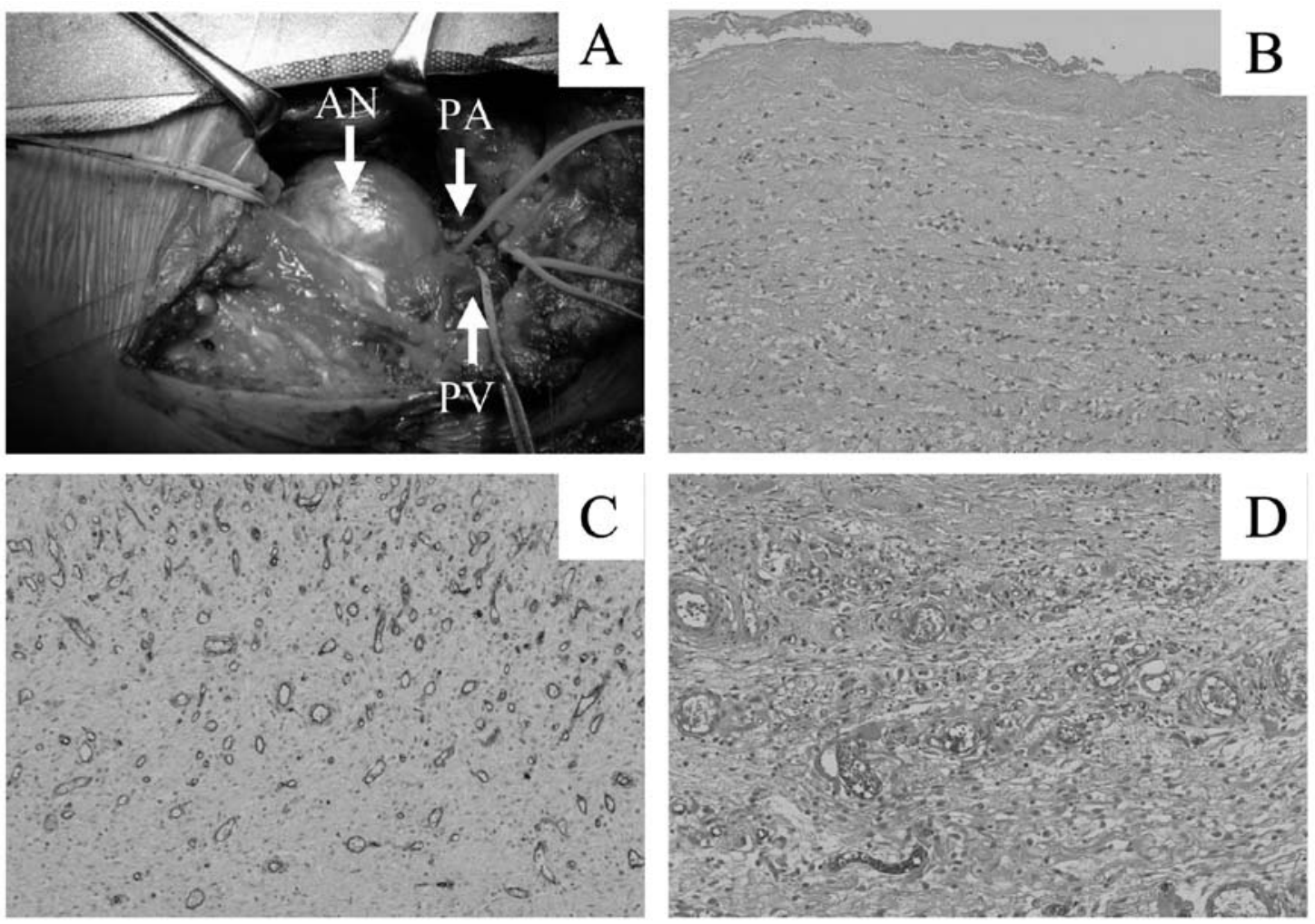

Fig. 3 Operative and histological findings.

A: Saccular aneurysm underlying expanded medial head of gastrocnemius muscle.

B: Hyalinized intimal thickening.

C: Proliferation of capillaries in media, showing by CD34 immunostain.

D: Marked proliferation of congestive capillaries with hemosiderin peposition in adeventitia.

PA: popliteal artery, PV: popliteal vein, AN: aneurysm

from the posterior surface of the medial epicondyle (Fig. 2). The popliteal aneurysm of $35 \times 45 \times 50 \mathrm{~mm}$ was surrounded by the expanded medial head of gastrocnemius muscle, and magnetic resonance angiography (MRA) showed stenosis of the artery just proximal to the popliteal aneurysm.

Resection of abnormal muscle and revascularization was elected for treatment of this popliteal aneurysm associated PAE syndrome. The popliteal fossa was explored through a posterior approach. The medial head of gastrocnemius muscle was expanded, and the lateral head of gastrocnemius muscle was compressed laterally. The accessory slip of gastrocnemius muscle that originated from the posterior surface of the medial condyle between the popliteal artery and the vein was excised, and the saccular aneurysm was exposed (Fig. 3A). Aneurysmotomy and interposition with reversed great saphenous vein was employed.

The postoperative course was uneventful. ABI on the right lower extremity was 1.13 in the normal position and 1.17 with passive dorsiflexion. Histologically, the intimal layer was hyalinized with segmented internal elastica, muscular media showed intensive proliferation of capillaries and moderate fibrosis, and the adeventitia disclosed marked proliferative congestive capillaries with hemosiderin deposition. The external elastica lamina was not seen (Figs. 3B, C, and D).

\section{DISCUSSION}

The anomalous relationship between the popliteal artery and the musculotendinous structures in patients with PAE results from abnormal development during the embryological phase. The development of the muscle through the popliteal fossa must complete before the popliteal artery is formed. If the artery is formed before muscle development is complete, the newly formed artery may be swept medial to the muscle, thereby resulting in PAE. The type of PAE is dependent on the extent and severity of this malformation. The present case can be classified as a type III congenital abnormality, because 
the popliteal artery was partially arrested as a result of development within the migrating muscle.)

PAE is uncommon but should be considered in younger athletic males with exercise-induced intermittent claudication. However, in the present case, PAE arose in the context of an asymptomatic popliteal aneurysm. In patients with PAE, popliteal aneurysms enlarged to $50 \mathrm{~mm}$ in diameter without ischemic symptoms are thought to be extremely rare. Rich et al. ${ }^{2}$ reported 9 cases of PAE over a 12-year period, and 2 of these 9 patients had popliteal aneurysm. In a review of PAE in 88 limbs (48 patients) by Levien et al., all cases demonstrated critical limb ischemia or typical claudication symptoms due to arterial occlusive pathology, and only 8 of 48 cases showed aneurysmal change. Also, di Marzo et al. ${ }^{3)}$ described 31 cases of PAE and reported that all cases demonstrated mild to severe ischemic symptom and that 2 of 31 cases showed aneurysmal change. Although diminishing ABI with passive dorsiflexion and stenosis of the popliteal artery just proximal to the aneurysm were noted, claudication symptom and peripheral thromboembolism were absent in the present case.

Popliteal aneurysm associated with PAE results from post-stenotic dilatation of the entrapped artery. Arterial wall histology in patients with PAE typically shows neovascularization and subsequent progressive fibrosis that occurs first in the adventitia with subsequent progression to involvement of the media. Thrombosis can result from replacement of the intimal region by fibrotic tissue. ${ }^{4)}$ The aneurysm of the present case is traumatic aneurysm in origin by minor mechanical stimuli for a long period. The localized diminution of medial smooth muscle cells with capillary proliferation mainly caused saccular type of aneurysm.

Arteriography has been the historical gold standard for the diagnosis of PAE, but recent studies suggest that MRI can accurately diagnose this phenomenon. ${ }^{5}$ In this case, CT was also helpful for diagnosis. Further, these latter modalities can provide information regarding morphological abnormalities between vessels and muscles, thereby assisting surgical planning. In the present case, imaging demonstrated an abnormal accessory muscle slip entrapping the artery, which was useful for surgery.

Adventitial cystic disease of the popliteal artery should be also considered as a possible diagnosis in case of limb ischemia or popliteal mass lesion in relative young patients. Angiographic appearance of popliteal adventitial cystic disease typically demonstrates a classical semilunar or crescentic luminal defect. In this case, CT demonstrated deviation of the proximal site of the popliteal artery as a result of compression by muscle and MRA showed stenosis of the artery just proximal to the popliteal aneurysm. Histology denied adventitial cystic disease and confirmed popliteal aneurysm associated with PAE.

The natural history of the popliteal artery with unrelieved compression was thought to be relatively progressive, and on this basis, surgery was advised whenever the diagnosis was confirmed. ${ }^{6}$ We performed resection of muscle slips and revascularization in this case because of huge aneurysmal change of popliteal artery. Aneurysmectomy and revascularization without muscle resection was difficult, because normal proximal popliteal artery could be hardly exposed due to muscle slips. Endovascular treatment was not applied because of stenotic lesion as well as aneurysm in the popliteal fossa. For patients with PAE, however, a reconstructive procedure results a relatively poor prognosis when compared with patients who require only simple muscle resection. ${ }^{7}$

Of the peripheral aneurysms, popliteal aneurysm is by far the most common, and the most common or almost exclusive cause is arteriosclerosis. Although PAE should be suspected in any young person under 30 age group who complains of intermittent claudication or who has developed an acute symptomatic occlusion of the popliteal artery, older patients with asymptomatic popliteal aneurysm should be also evaluated for the possibility of PAE.

\section{REFERENCES}

1) Levien LJ, Veller MG. Popliteal artery entrapment syndrome: more common than previously recognized. J Vasc Surg. 1999; 30: 587-98.

2) Rich NM, Collins GJ Jr, McDonald PT, Kozloff L, Clagett GP, Collins JT. Popliteal vascular entrapment. Arch Surg 1979; 114: 1377-84.

3) di Marzo L, Cavallaro A, Sciacca V, Mingoli A, Tamburelli A. Surgical treatment of popliteal artery entrapment syndrome: a ten-year experience. Eur J Vasc Surg 1991; 5: 59-64.

4) Levien LJ. Popliteal artery thrombosis caused by popliteal entrapment syndrome. In: Greenhalgh RM, Powell JT, editors. Inflammatory and thrombotic problems in vascular surgery. London: W.B. Saunders Co Ltd, 1997: 159-68.

5) Fujiwara H, Sugano T, Fujii N. Popliteal artery entrapment syndrome: accurate morphological diagnosis utilizing MRI. J Cardiovasc Surg. 1992; 33: 160-2.

6) Fowl RJ, Kempczinski RF, Whelan TJ. Popliteal artery entrapment. In: Rutherford RB, editor. Vascular surgery. 4th ed. Philadelphia: W.B. Saunders Co,1995: 889-94.

7) di Marzo L, Cavallaro A, Sciacca V, Mingoli A, Stipa S. Natural history of entrapment of the popliteal artery. J Am Coll Surg. 1994; 178: 553-6. 